\title{
Electronic Mapping of Molecular Orbitals at the Molecule-Metal Interface
}

\author{
M. C. Lennartz, V. Caciuc, N. Atodiresei, S. Karthäuser,* and S. Blügel \\ Institut für Festkörperforschung and JARA, Forschungszentrum Jülich GmbH, 52425 Jülich, Germany
}

(Received 21 April 2010; published 4 August 2010)

\begin{abstract}
The molecule-metal interface formed by pyridine-2,5-dicarboxylic acid chemically bonded to the $\mathrm{Cu}$ (110) surface is investigated by scanning tunneling microscopy and first-principles calculations. Our current-voltage spectroscopy studies reveal an electronic mapping of molecular orbitals as a function of tip position. By combining experimental and theoretical investigations, individual molecular orbitals are characterized by their energy and spatial distribution. The importance of adsorption geometries and conformational changes on the electron transport properties is highlighted.
\end{abstract}

In the past decade, we have witnessed significant progress in integrating organic molecules in functional molecular devices like single molecule diodes $[1,2]$ or in organic field-effect transistors [3,4]. To improve the functionality of such molecular electronic devices, an essential prerequisite is to gain a substantial understanding of the electronic structure of molecule-surface interfaces near the Fermi level that ultimately controls the performance of such a device [5-7].

In this context, the precise energetic alignment of the molecular orbitals with respect to the Fermi level of the substrate, in particular, that of the highest occupied molecular orbitals and the lowest unoccupied molecular orbitals, is a key component of the electronic structure of the molecule-surface system under consideration. Therefore, in this Letter we present a detailed electronic mapping of molecular orbitals (MOs) by distance-dependent currentvoltage $(I-V)$ spectroscopy. Combining the spatially resolved scanning tunneling spectroscopy (STS) results with density functional theory (DFT) investigations, we show how to identify the electronic structure of a molecule chemically bonded to a metallic surface. As a model system we investigate the adsorption of the pyridine-2,5dicarboxylic acid $\left(\mathrm{PyDCAH}_{2}\right)$ on $\mathrm{Cu}(110)$.

Our $a b$ initio total-energy calculations have been performed in the framework of DFT [8] by using the PerdewBurke-Ernzerhof [9] exchange-correlation energy functional as implemented in the VASP code $[10,11]$. A detailed description is available in the supplementary material [12]. We first note that, in the case of the $\mathrm{PyDCAH}_{2}$ molecule, which adsorbs on the $\mathrm{Cu}(110)$ surface under deprotonation of one carboxyl group, forming PyDCAH, several different adsorption geometries must be taken into account due to the presence of a nitrogen atom in the aromatic ring. Therefore, we differentiate in the following between $\mathrm{C}_{7} \mathrm{H}_{4} \mathrm{~N}^{(2)} \mathrm{O}_{4}$, describing a PyDCAH molecule with the nitrogen on position two in the ring (counting the ring atoms from the carbon atom located at the bonded carboxylate unit), and $\mathrm{C}_{7} \mathrm{H}_{4} \mathrm{~N}^{(3)} \mathrm{O}_{4}$, denoted as configuration $\{1\}$ and $\{2\}$, respectively. Experimentally, there is no possibil- ity to influence the orientation of the molecule during the adsorption process or to monitor topographically which configuration is preferentially adsorbed. If the PyDCAH molecule adsorbs in configuration $\{2\}$ on the surface, the orientation of the $\mathrm{COOH}$ top group becomes important and three conformations are conceivable [see insets in Fig. 1(a)]. We note that the conformers $\{2 a\}$ and $\{2 b\}$ differ through a rotation of the $\mathrm{COOH}$ group around the $\mathrm{C}-\mathrm{C}$ bond between the ring and carboxylic group. On the other hand, the conformers $\{2 b\}$ and $\{2 c\}$ differ through a rotation of the hydroxyl $(\mathrm{OH})$ group around the $\mathrm{C}-\mathrm{O}$ bond within the carboxylic group. Comparing the different adsorption geometries, in terms of their adsorption energies, leads to a PyDCAH molecule adsorbed in conformation $\{2 c\}$ onto the surface as the ground state. It exhibits a hydrogen bond between the carboxylic group and the nitrogen atom of the aromatic ring. However, the other metastable conformations without a hydrogen bond are with $120(\{2 a\})$ and $190 \mathrm{meV}(\{2 b\})$ slightly higher in energy [13], with respect to the ground state configuration $\{2 c\}$. Furthermore, the calculated adsorption energies of the $\mathrm{C}_{7} \mathrm{H}_{4} \mathrm{~N}^{(2)} \mathrm{O}_{4}(\{1\})$ and $\mathrm{C}_{7} \mathrm{H}_{4} \mathrm{~N}^{(3)} \mathrm{O}_{4}(\{2 a\})$ configurations are practically identical and are similar to those reported for other molecules that adsorb via carboxylate group on the $\mathrm{Cu}(110)$ surface [14-16]. All rotation barriers for the rotations described above and the relative energies of the different adsorption geometries are available as supplementary material [12].

In Fig. 1(a), we present the local density of states (LDOS) calculated for the four possible adsorption geometries related to the position of the nitrogen atom and the $\mathrm{H}$ atom of the carboxylic group. In particular, we focus on the molecule-surface electronic states at the molecular side which have a predominant $\sigma$ (i.e., in-plane) or $\pi$ (i.e., out-of-plane) contribution with respect to the molecular plane. We exemplarily plot the spatial distribution of the charge density in a specific energy range associated with the molecular LDOS for the conformation $\{2 a\}$ in Fig. 1(b) and for the configuration $\{2 c\}$ in Ref. [12]. Close to the Fermi level, the LDOS shows three occupied $\sigma$ orbitals 


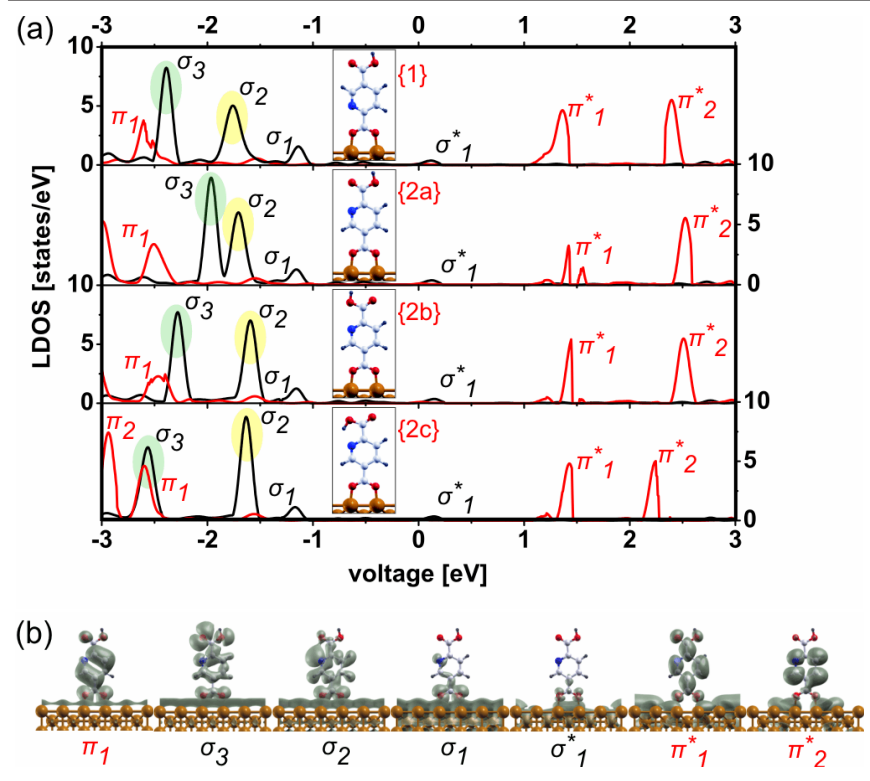

FIG. 1 (color online). (a) Calculated LDOS of a PyDCAH molecule chemically bonded to the $\mathrm{Cu}(110)$ surface in four possible configurations: $\mathrm{C}_{7} \mathrm{H}_{4} \mathrm{~N}^{(2)} \mathrm{O}_{4}, \mathrm{C}_{7} \mathrm{H}_{4} \mathrm{~N}^{(3)} \mathrm{O}_{4}\{2 a\}$ with the $\mathrm{H}$ atom of the carboxylic group pointing up, $\mathrm{C}_{7} \mathrm{H}_{4} \mathrm{~N}^{(3)} \mathrm{O}_{4}$ $\{2 b\}$ with the $\mathrm{H}$ atom pointing up above the nitrogen atom, and with the $\mathrm{H}$ atom pointing down $\{2 c\}$ towards the $\mathrm{N}$ atom. The red line represents the states with a $\pi$-type character, and the black line the states with the $\sigma$-type character. (b) Charge density plots of the electronic states of $\mathrm{C}_{7} \mathrm{H}_{4} \mathrm{~N}^{(3)} \mathrm{O}_{4}\{2 a\}$ in a specific energy range corresponding to the LDOS depicted above.

which can be related, with the help of the charge density plots, to the carboxylic top group $\left(\sigma_{3}\right)$, the nitrogen atom in the ring $\left(\sigma_{2}\right)$, and the binding carboxylate group $\left(\sigma_{1}\right)$. For the bonding $\sigma_{1}$ and the antibonding $\sigma_{1}^{*}$ states, we note that the charge density is mainly localized on the carboxylate group $\left(\mathrm{COO}^{-}\right)$leaving the top carboxylic group $(\mathrm{COOH})$ at the vacuum interface with no charge contribution. This charge density localized between the bonding carboxylate functionality and the copper surface illustrates the interaction of the molecular orbitals with the $d$-band electrons of the copper. Furthermore, the LDOS calculations show three $\pi$ orbitals which are all predominantly located at the pyridine ring. From the electronic point of view, the presence of nitrogen in conjugated heterocyclic molecules lowers their $\pi$-orbital energies while some of the $\sigma$ orbitals are pushed to higher binding energies [17-19]. This feature can be seen for all adsorption geometries shown in Fig. 1(a). One can also observe that the hydrogen bond (configuration $\{2 c\}$ ) shifts the $\sigma_{3}$ orbital to lower binding energies. As a general observation, the carboxylate group of PyDCAH is involved in all bonding molecular states like $\pi_{1}$ and $\sigma_{3}$ to $\sigma_{1}$.

STM investigations were performed on self-assembled monolayers of PyDCAH on $\mathrm{Cu}(110)$ single crystals. The experimental details are available in Ref. [12]. Images were obtained in constant-current mode, while spectroscopy data are current-voltage characteristics with the volt- age referred to the sample. Our STM and DFT investigations on the structure show that the bonding mechanism of $\mathrm{PyDCAH}_{2}$ is similar to that of other carboxylic acids on $\mathrm{Cu}(110)[16,20-22]$. The molecules bind chemically under deprotonation of one carboxyl group with each of the oxygen atoms chemisorbed on short bridge sites of the outermost copper layer, forming rows in the [001] direction of the substrate. The pyridine rings are arranged in the same plane as the carboxylate moiety, perpendicular to the surface. Our previous study has shown that no significant intermolecular interaction between the adsorbed molecules occurs even at high coverages [16], and therefore the LDOS calculations of single molecules adsorbed on $\mathrm{Cu}(110)$ provide an adequate description.

Before starting spectroscopic measurements, and after a series of measurements, topographic imaging of the sample was performed. An example of a high resolution STM topography image is shown in Fig. 2. The PyDCAH molecules are ordered in a close-packed structure of standing up molecules. The STM tip was positioned above a molecule and then the STM was put into spectroscopy mode. A series of $I-V$ measurements was started with the tip position far away from the molecule. Here the set point tunneling current was $I_{\text {Set }}=0.1 \mathrm{nA}$ at a sample bias voltage of $V_{\text {Set }}=-2.0 \mathrm{~V}$. After the feedback loop had been turned off, the bias voltage was increased from -3.0 to $+3.0 \mathrm{~V}$, while recording the resulting current at steps of $0.01 \mathrm{~V}$. After each voltage sweep, the feedback loop was turned on again and the tip height was readjusted before starting again. After ten sweeps, $I_{\text {Set }}$ was increased by $0.1 \mathrm{nA}$, while the sample bias voltage was kept constant and a new series of sweeps was started. This procedure was repeated until $I_{\text {Set }}=0.6 \mathrm{nA}$ was reached. For higher currents a significant change in the topographical image was visible, presumably due to the destruction of the molecular layer. The same procedure was repeated several times and with different tips. In this way an unambiguous assignment of the $I-V$ curves to the electronic structure of the PyDCAH-Cu system is possible. All spectroscopy data shown here were averaged over ten curves and compared with the data obtained for various molecules spread over

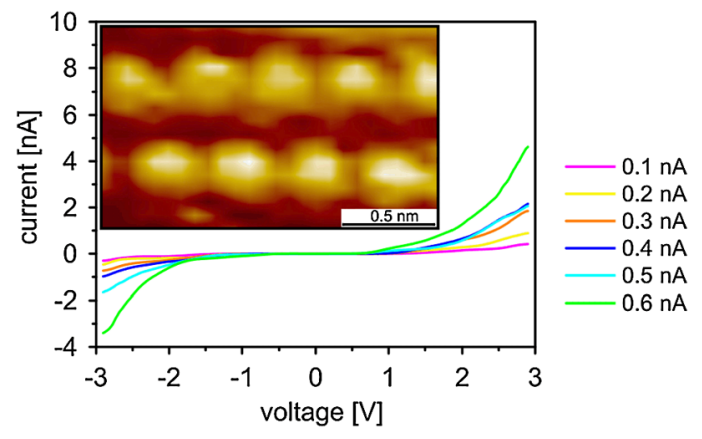

FIG. 2 (color online). $\quad I-V$ spectra of a PyDCAH molecule on $\mathrm{Cu}(110)$ in dependence of the set point current and high resolution STM image of PyDCAH molecules ordered in a closepacked structure of standing up molecules. 
the samples. In Fig. 2, the results of a series of currentvoltage spectroscopy measurements at various tip-sample distances are illustrated.

For an interpretation of the spectroscopic data, $d I / d V$ curves were calculated from the $I-V$ data. We forbear from plotting the normalized differential conductance in this work because we focus on the appearance and disappearance of peaks in dependence on the tip-sample distance. The $d I / d V$ curves can be compared to the LDOS calculated for the adsorbed PyDCAH on $\mathrm{Cu}(110)$ by DFT. In a first step, all measured $d I / d V$ curves of the PyDCAH molecule were plotted separately, and a first finding is that, starting from a set point current of $0.1 \mathrm{nA}$ and going to $0.3 \mathrm{nA}$, an accretion of defined peaks in the $d I / d V$ spectra can be measured. The peaks do not change their shape or their energy. This means that at this large tipsample distance the STM detects the same molecular orbitals. Further increasing $I_{\mathrm{Set}}$ causes changes in the number of monitored peaks as well as changes in their intensity and energetic position until at $0.7 \mathrm{nA}$ the tip causes irreversible changes in the molecular layer.

We first focus on the unoccupied molecular orbitals, e.g., $\sigma_{1}^{*}, \pi_{1}^{*}$, and $\pi_{2}^{*}$, and analyze their behavior in dependence of the set point current. At $0.3 \mathrm{nA}$ a first peak at $+1.5 \mathrm{~V}$ and at $0.4 \mathrm{nA}$ the appearance of a second peak at around $+2.3 \mathrm{~V}$ are observed. With increasing current, both peaks then shift to lower energies whereas $\sigma_{1}^{*}$ is not detectable. By combining the experimental peak energies with the LDOS calculations for the four possible adsorption geometries of the PyDCAH molecules [Fig. 1(a)], we conclude that the first appearing peak is related to the $\pi_{1}^{*}$ orbital and the second one to the $\pi_{2}^{*}$ orbital. Comparing the isosurface plots [Fig. 1(b)] of these two orbitals reveals that the $\pi_{1}^{*}$ orbital has an extension at the carboxylic top group, whereas the $\pi_{2}^{*}$ orbital is located mostly at the aromatic ring. Accordingly, if the tip is moved towards the sample, first the $\pi_{1}^{*}$ orbital and then the $\pi_{2}^{*}$ orbital should be detected, as predicted by theory. At closer distances the electric field of the tip causes the shift of these orbitals to lower energies as reported also for other $\pi$-conjugated systems $[23,24]$. Furthermore, the isosurface plots show a location of the $\sigma_{1}^{*}$ orbital direct at the copper surface, explaining that no detection is possible within the current window of experiment. From the analysis of the unoccupied MOs, no differentiation between different adsorption geometries can be made because all LDOS fit more or less to the experimental $d I / d V$ curves, visible on the righthand sides of the spectra in Fig. 3.

The occupied molecular orbitals, e.g., $\pi_{1}, \sigma_{1}, \sigma_{2}$, and especially $\sigma_{3}$, show a more complex behavior depending on the set point current. At $I_{\text {Set }}=0.3 \mathrm{nA}$, two peaks are detectable in the experimental $d I / d V$ spectrum beside the unoccupied $\pi_{1}^{*}$-orbital peak. The first peak appears as a sharp peak at around $-2.6 \mathrm{~V}$, whereas the second one, at around $-1.75 \mathrm{~V}$, is broad and could be caused by monitoring two molecular orbitals. Comparing the isosurface

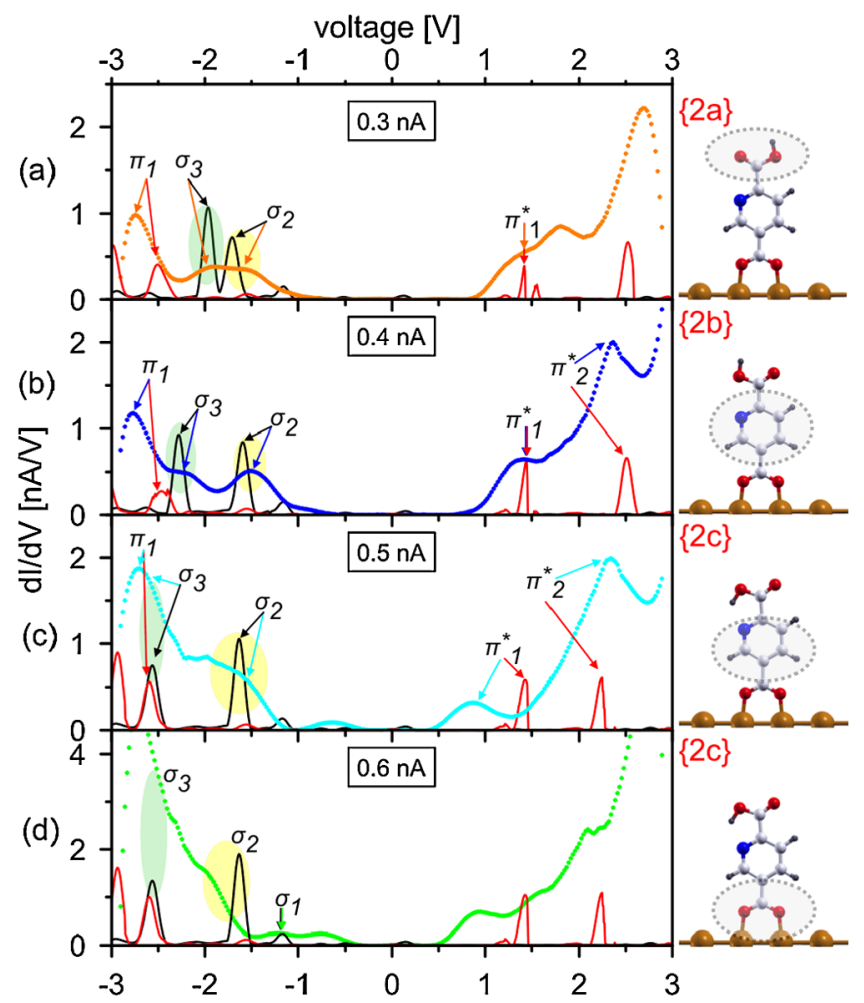

FIG. 3 (color online). Experimental $d I / d V$ spectra acquired over PyDCAH on $\mathrm{Cu}(110)$ with varying current set points at $V_{\text {Set }}=-2.0 \mathrm{~V}$ and calculated LDOS with the $\sigma$ and $\pi$ orbitals marked in black and red, respectively. With increasing set point current (a)-(d), the $\sigma_{3}$ orbitals (marked in light green) change their energetic position relative to $\sigma_{2}$ and $\pi_{1}$ indicating configurational changes. Outlined in the schematic plots on the right are the molecular parts detected as a function of tip height.

plots leads to an expected appearance of the $\sigma_{3}$ orbital, of the $\sigma_{2}$ orbital in lower intensity, and of the $\pi_{1}$ orbital at this tip-sample separation. Analysis of the top-group-related $\sigma_{3}$ peak shows that the experimental data do not fit to geometry $\{1\}$ and $\{2 b\}$. These geometries can be excluded as the starting configuration of the PyDCAH molecules under investigation. With this we can conclude that the $\mathrm{PyDCAH}_{2}$ molecules are not adsorbed in configuration $\{1\}$. The two remaining conformations are both consistent with the experimental data [Fig. 3(a)]. In the case of conformation $\{2 a\}$ both orbitals $\sigma_{3}$ and $\sigma_{2}$ can be assigned to the broad peak, whereas in conformation $\{2 c\}$ the energies of $\sigma_{3}$ and $\pi_{1}$ are the same. At this set point, only the orbitals located in the upper part of the PyDCAH molecule are monitored. Decreasing the tip-sample distance, i.e., increasing $I_{\text {Set }}$ to $0.4 \mathrm{nA}$, results in $d I / d V$ spectra plotted in Fig. 3(b). Three occupied MOs are visible in the experimental spectrum. The $\pi_{1}$ orbital at high negative energy stays constant, whereas the $\sigma_{2}$ peak gets sharper at slightly lower negative energy. In between these peaks, a new one can be detected at around $-2.2 \mathrm{~V}$. Comparing again with the LDOS calculations, we conclude that conformation $\{2 a\}$ is not compatible with these measured $d I / d V$ curves whereas conformation $\{2 b\}$ fits best. The middle peak is 
identified to be the $\sigma_{3}$ orbital. In the case of molecules like PyDCAH, a changing of conformations in dependence of the set point current is possible because the carboxylic top group can rotate around the $\mathrm{C}-\mathrm{C}$ bond. Thus, experimental data point to a rotation in the presence of the applied electric field from conformation $\{2 a\}$ to $\{2 b\}$. The $d I / d V$ spectrum at $I_{\text {Set }}=0.5 \mathrm{nA}[$ Fig. 3(c)] shows a convergence of the $\pi_{1}$ and the $\sigma_{3}$ peak, and they are recorded with high intensity at $-2.7 \mathrm{~V}$. The conformation which fits best to the experimental data is here conformation $\{2 c\}$. This again points to a rotation, in the way that the $\mathrm{H}$ atom of the top carboxylic group rotates around the $\mathrm{C}-\mathrm{O}$ bond. Further lowering the tip position $(0.6 \mathrm{nA})$ results in $d I / d V$ curves reflecting also the molecular orbital located at the binding carboxylate group, i.e., $\sigma_{1}$ [Fig. 3(d)]. This $\sigma$-type orbital is localized near the $\mathrm{Cu}(110)$ substrate and has an energy close to the Fermi energy.

Compiling the results of the detailed single set point analysis concerning the adsorption geometries shows that two interpretations are possible. A first possibility is that the investigated $\mathrm{PyDCAH}_{2}$ molecules are adsorbed in the stable conformation $\{2 c\}$, with a hydrogen bond between the carboxylate top group and the nitrogen. In this case, the $d I / d V$ spectra at $I_{\text {Set }}=0.4 \mathrm{nA}$ cannot be fully explained by the present data. The second possibility is a combination of different conformations which are converted by rotations of the atoms of the carboxylic top group. Starting with conformation $\{2 a\}$, the top carboxylic group rotates with increasing electric field, resulting in conformation $\{2 b\}$ at $I_{\text {Set }}=0.4 \mathrm{nA}$. The hydrogen atom is then located above the nitrogen, and due to interactions, caused by the increasing electric field, the $\mathrm{H}$ atom of the carboxylic top group flips around the $\mathrm{C}-\mathrm{O}$ bond. The molecule ends in conformation $\{2 c\}$ at $I_{\mathrm{Set}}=0.5 \mathrm{nA}$. Recent publications have shown that a configurational change of a molecule on a metal surface can be imaged by STM $[25,26]$ and that a rotation of molecules in an increasing electric field can be monitored by a sequence of STM images [27]. Consequently, the change of electronic properties related to different adsorption geometries can be monitored by STS, as reported in this work.

In summary, we prove that distance-dependent $I-V$ spectroscopy can not only map the molecular orbital energies of the PyDCAH-Cu(110) system but also the spatial distribution of the different orbitals. While delocalized states like the unoccupied $\pi$ orbitals are monitored over a range of set points, the in-plane localized $\sigma$ orbitals are observed at specific set points. Thus, combining DFT calculations and distance-dependent STS enables an electronic mapping of molecules connected to a metal surface. Furthermore, the LDOS calculated for PyDCAH bonded to a metallic surface is used as a fingerprint for analyzing their adsorption geometries.

This work was supported by the DFG Priority Program SPP1243. The theoretical calculations have been performed at the Jülich Supercomputing Centre. The authors thank P. Lazic for discussions. *s.karthaeuser@fz-juelich.de

[1] I. I. Oleynik, M. A. Kozhushner, V.S. Posvyanskii, and L. Yu, Phys. Rev. Lett. 96, 096803 (2006).

[2] I. Díez-Pérez, J. Hihath, Y. Lee, L. Yu, L. Adamska, M. A. Kozhushner, I. I. Oleynik, and N. Tao, Nature Chem. 1, 635 (2009).

[3] C.D. Dimitrakopoulos, S. Purushothaman, J. Kymissis, A. Callegari, and J. M. Shaw, Science 283, 822 (1999).

[4] V. C. Sundar, J. Zaumseil, V. Podzorov, E. Menard, R. L. Willett, T. Someya, M. Gershenson, and J. Rogers, Science 303, 1644 (2004).

[5] H. Ishii, K. Sugiyama, E. Ito, and K. Seki, Adv. Mater. 11, 605 (1999).

[6] G. Heimel, L. Romaner, E. Zojer, and J.-L. Brédas, Nano Lett. 7, 932 (2007)

[7] L. Vitali, G. Levita, R. Ohmann, A. Comisso, A. D. Vita, and K. Kern, Nature Mater. 9, 320 (2010).

[8] P. Hohenberg and W. Kohn, Phys. Rev. 136, B864 (1964).

[9] J. P. Perdew, K. Burke, and M. Ernzerhof, Phys. Rev. Lett. 77, 3865 (1996).

[10] G. Kresse and J. Hafner, Phys. Rev. B 47, 558 (1993).

[11] G. Kresse and J. Furthmüller, Phys. Rev. B 54, 11169 (1996).

[12] See supplementary material at http://link.aps.org/ supplemental/10.1103/PhysRevLett.105.066801 for theoretical and experimental methods.

[13] The energy differences between the configuration $\{2 a\}$ and $\{2 c\}$ changes from 120 to $134 \mathrm{meV}$ when increasing the plane-wave energy cutoff from 500 to $700 \mathrm{eV}$. Therefore, our estimated error bar is of $14 \mathrm{meV}$.

[14] N. Atodiresei, K. Schroeder, and S. Blügel, Phys. Rev. B 75, 115407 (2007).

[15] N. Atodiresei, V. Caciuc, K. Schroeder, and S. Blügel, Phys. Rev. B 76, 115433 (2007).

[16] M. C. Lennartz, N. Atodiresei, L. Müller-Meskamp, S. Karthäuser, R. Waser, and S. Blügel, Langmuir 25, 856 (2009).

[17] I. Fleming, Frontier Orbitals and Organic Chemical Reactions (Wiley, New York, 1978).

[18] G. Zeiss and M. Whitehead, J. Chem. Soc. A 1971, 1727 (1971).

[19] N. Atodiresei, V. Caciuc, P. Lazic, and S. Blügel, Phys. Rev. Lett. 102, 136809 (2009).

[20] B. G. Frederick, F.M. Leibsle, S. Haq, and N. V. Richardson, Surf. Rev. Lett. 3, 1523 (1996).

[21] J. Lee, O. Kuzmych, and J. T. Y., Jr., Surf. Sci. 582, 117 (2005).

[22] O. Karis, J. Hasselstroem, N. Wassdahl, M. Weinelt, A. Nilsson, M. Nyberg, L. G. M. Petterson, J. Stoehr, and M. G. Samant, J. Chem. Phys. 112, 8146 (2000).

[23] Y. Li, J. Zhao, X. Yin, and G. Yin, J. Phys. Chem. A 110, 11130 (2006).

[24] C. Kim, B. Kim, S. M. Lee, C. Jo, and Y. H. Lee, Phys. Rev. B 65, 165418 (2002).

[25] J. Repp, G. Meyer, S. Paavilainen, F. E. Olsson, and M. Persson, Science 312, 1196 (2006).

[26] V. Simic-Milosevic, J. Meyer, and K. Morgenstern, Angew. Chem., Int. Ed. 48, 4061 (2009).

[27] H. L. Tierney, A.E. Baber, and E.C. H. Sykes, J. Phys. Chem. C 113, 10913 (2009). 